\title{
ATM and ATR INFLUENCE MEIOTIC CROSSOVER FORMATION THROUGH ANTAGONISTIC and OVERLAPPING FUNCTIONS in C. elegans
}

\section{Wei $\mathrm{Li}^{1,2}$ and Judith Yanowitz ${ }^{1, *}$}

${ }^{1}$ Magee-Womens Research Institute, Department of Obstetrics, Gynecology, and Reproductive Sciences, University of Pittsburgh School of Medicine, 204 Craft Avenue

Pittsburgh, PA 15213, USA. ${ }^{2}$ Tsinghua University MD Program, School of Medicine, Tsinghua University, Haidian District, Beijing 100084, P.R.China

*Corresponding author 
bioRxiv preprint doi: https://doi.org/10.1101/604827; this version posted April 10 2019. The copyright holder for this preprint (which was not certified by peer review) is the author/funder, who has granted bioRxiv a license to display the preprint in perpetuity. It is made available under aCC-BY-NC 4.0 International license.

\title{
Short title: Antagonism and synergism of ATM and ATR
}

\author{
Key Words: \\ ATM \\ ATR \\ Meiosis \\ C. elegans \\ DSB repair
}

\section{*Corresponding author}

Judith Yanowitz

Magee-Womens Research Institute

204 Craft Avenue Rm

Pittsburgh, PA 15213

yanowitzj1@mwri.magee.edu

412.641.7843 


\section{Article Summary}

Crossovers formed during meiosis connect homologs and properly align them for cell division. The central importance of crossovers is underscored by the existence of extensive regulatory processes that ensures the proper execution of these events. This paper explores the evolutionary conserved roles of the central DNA damage response kinases, ATM and ATR, in crossover formation. The authors show that these kinases function together as rheostats to promote timely formation of crossovers on all chromosomes but to limit extensive DNA damage. This work provides a platform for identifying conserved meiotic targets of ATM and ATR that affect fertility across species. 


\begin{abstract}
During meiosis, formation of double-strand breaks (DSBs) and repair by homologous recombination between homologs creates crossovers (COs) that facilitate chromosome segregation. $\mathrm{CO}$ formation is tightly regulated to ensure the integrity of this process. The DNA damage response kinases, Ataxiatelangiectasia mutated (ATM) and RAD3-related (ATR) have emerged as key regulators of CO formation in yeast, flies, and mice, influencing DSB formation, repair pathway choice, and cell cycle progression. The molecular networks that ATM and ATR influence during meiosis are still being resolved in other organisms. Here we show that Caenorhabditis elegans ATM and ATR homologs, ATM-1 and ATL-1 respectively, act at multiple steps in CO formation to ultimately ensure that COs are formed on all chromosomes. We show a role for ATM-1 in regulating the choice of repair template, biasing use of the homologous chromosome instead of the sister chromatid. Our data suggests a model in which ATM-1 and ATL-1 have antagonistic roles in very early repair processing, but are redundantly required for accumulation of the RAD-51 recombinase at DSB sites. We propose that these features of ATM-1 and ATL-1 ensure both CO formation on all chromosomes and accurate repair of additional DSBs.
\end{abstract}




\section{INTRODUCTION}

Crossover recombination, the exchange of DNA between homologous chromosomes, occurs in meiosis I and is a key step to ensure that chromosomes are segregated properly. Many factors contribute to the recombination outcome, including the number of double-strand breaks (DSBs) made during meiosis, their distribution, and the repair pathway chosen following the formation of DSBs. The formation and repair of DSBs and the conversion of some of these into crossovers (COs) is a highly regulated process that must be tightly controlled to ensure the proper subsequent segregation of chromosomes. Mechanisms have evolved to tune DSB numbers to species-specific levels (GRAY et al. 2013) and also to channel a limited number of DSBs into COs (MARTINI et al. 2006).

The DNA damage response (DDR) kinases ATM/Tel1 (ataxia-telangiectasia mutated) and ATR/Mec1 (ataxia-telangiectasia and RAD-3 related) have emerged as key conserved players in CO homeostasis (reviewed in (MACQUEEN AND HochWAGEN 2011; COOPER et al. 2014)). ATM mutations lead to severe meiotic defects and infertility in mice, yeast and flies (BARLOW et al. 1996; XU et al. 1996; BARLOW et al. 1998). ATM both downregulates Spo11-mediated DSBs (JOYCE et al. 2011; LANGE et al. 2011; ZHANG et al. 2011; CARBALLO et al. 2013; GARCIA et al. 2015) and affects their distribution (ZHANG et al. 2011; ANDERSON et al. 2015; GARCIA et al. 2015). It also biases use of the homolog as a repair template (inter-homolog homologous recombination (IH-HR)) versus the sister chromatid (intersister (IS-HR)). ATR, by contrast, positively regulates DSB formation (GRAY et al. 2013). Together ATM and ATR establish a regulatory feedback through the phosphorylation of components of the DSB machinery and chromatin axes (COOPER et al. 2014). Although yeast ATM and ATR (Tel1 and Mec1, respectively) seem to function antagonistically to enforce DSB homeostasis, the interplay between the two genes and their functions in $\mathrm{CO}$ formation are more complex. For example, research in yeast supports that there is a DSB threshold above which Tel1 plays a role (with Pch2 and Mec1) in homolog bias, while with low-abundance DSBs, Tel1 promotes resection (JOSHI et al. 2015; MIMITOU et al. 2017). Thus, further insights into the functional consequences of ATM/ATR loss on CO formation through the analysis of additional model systems may help provide new insights into ATM/ATR function.

In Caenorhabditis elegans, the orthologs of ATM and ATR, ATM-1 and ATL-1 (ATM-like), are required for genome stability (JONES et al. 2012). In addition, more RAD-51 foci were observed in meiotic cells in atm-1 mutants compared to wild type, a result that was consistent with a conserved role 
in DSB inhibition (CHECCHI et al. 2014). However, further information about the role of ATM-1 in DSB and CO formation remains unknown. Loss of atm-1 function has been reported to lead to a mild increase in meiotic nondisjunction suggesting a more complex relationship between DSB formation and $\mathrm{CO}$ induction. Because most chromosomes receive a single $\mathrm{CO}$ each meiosis (high $\mathrm{CO}$ interference) and because only a single $\mathrm{CO}$ pathway has been identified in C. elegans, we reasoned that the worm offered an opportunity to investigate both conserved features of ATM and ATR functions in meiosis and to dissect out their contributions to unique aspects of $\mathrm{CO}$ control that are harder to examine in other systems. Here we show that $C$. elegans atm-1 and atl-1 act at multiple steps in CO formation to ultimately ensure that COs are formed on all chromosomes.

\section{MATERIALS AND METHODS}

\section{Genetics and worm handling}

All strains were grown and maintained at $20^{\circ}$ on standard media (BRENNER 1974). Mutant strains used in this study were: LGI atm-1(gk186), rad-54(ok615); LGII $d s b-2(m e 96)$, smc-5(ok2421), ); meIs8[unc-119(+) pie-1promoter::gfp::cosa-1]; LGIII brc-1(tm1145), dpy-18(e364), unc-64(e246), dpy-1(e1), lon-1(e185); LGIV spo-11(me44), dsb-1(we11); LGV him-5(ok1896), syp-1(me17), atl1(tm853). atm-1 (gk186) is a deletion allele that removed upstream promoter sequences and half of the 5' coding sequence; it is a presumptive null. atl-1(tm853) is an $700 \mathrm{bp}$ deletion in the coding sequence that is a strong loss-of-function or null. Some strains were provided by the Caenorhabditis Genetics Center that is funded by National Institutes of Health - Office of Research Infrastructure Programs (P40 OD010440). Double, triple and quadruple mutants were generated using standard genetic techniques with PCR verification of genotypes and are listed in Supplementary Table S1.

\section{Immunofluorescence}

Adults worms were dissected in $1 \mathrm{X}$ sperm salts with $1 \mathrm{mM}$ levamisole and fixed in $2 \%$ paraformaldehyde/1 X PBS for $5 \mathrm{~min}$ in a humid chamber. Slides were then freeze-cracked and immersed in $100 \%$ ethanol for 2 min followed by 5 seconds in acetone. Slides were then washed in PBSTB (1 X PBS with 0.1\% Tween and 0.1\% bovine serum albumin (BSA)) and incubated overnight at $4^{\circ}$ in primary antibody (rabbit anti-RAD-51, 1:30000, gift from S. Smolikove, rabbit anti-DSB-2, 1:20000, gift from A. Villeneuve) diluted in PBSTB. The next day, slides were washed $3 \times$ PBSTB and incubated in secondary antibody ( $\alpha$-rabbit Alexa 568, 1:2000) for 4 hours at room temperature in the dark. Then slides were washed 2 x 10 minutes, and stained 1 x 10 min with DAPI $(10 \mathrm{mg} / \mathrm{ml}$ stock 
diluted 1:50000 in 1 X PBS). Slides were mounted in Prolong Gold with DAPI and put in the dark to dry overnight before imaging.

\section{Analysis of RAD-51 foci}

3D images of the whole germ lines were taken using a Nikon A1r confocal microscope and analyzed using Volocity 3D software (PerkinElmer). For wild type and atm-1, him-5, dsb-2, atm-1; him-5 and atm-1;dsb-2 mutants, as well as irradiated and non-irradiated spo-11 mutants and atm-1;spo-11, we divided the pachytene region into six zones and counted RAD-51 foci in every nucleus for a minimum of three germ lines/ genotype. For rad-54; him-5 mutants and atm-1;rad-54; him-5 mutants, we counted the RAD-51 foci in late pachytene nuclei for at least three germ lines/ genotype. For analysis of atl-1 mutants (Figure 7,8), the transition zone (TZ) was included and the pachytene zones were binned into 3 regions $(1+2)$ /zone $(3+4)$ /zone $(5+6)$ shown in Figure $2 \mathrm{~A})$. Three gonad arms were analyzed/ genotype.

\section{Irradiation}

Day 1 adult worms were exposed to $\gamma$-irradiation from a ${ }^{137} \mathrm{Cs}$ source (Gammacell1000 Elite, Nordion International Inc.). Dosages are described in text. For analysis of diakinesis stage nuclei post-IR, animals were fixed and stained $27 \mathrm{hrs}$ post-IR(MCCLENDON et al. 2016). For the worms used for RAD51 staining for time course analysis, we stained them 1, 2, 4, and 8 hrs post-IR.

\section{Recombination analysis}

Recombination rates between $d p y-18$ and unc- 64 or $d p y-1$ and lon- 1 performed by crossing the marker mutations into the respective genetic background and assaying for . Dpy non-Unc and Unc-nonDpy progeny from $d p y-18 u n c-64 /+$ parents or Lon non-Dpy (Dpy is epistatic to Lon, so cannot be assessed for recombination). More than 1000 progeny for each phenotype were analyzed and the recombination rate was calculated based on prior results(BRENNER 1974).

\section{Analysis of GFP::COSA-1 foci}

Day 1 adult worms were dissected in $2 \mathrm{x}$ sperm salts as described above. Slides were immediately freeze-cracked and immersed in 100\% ethanol for $10 \mathrm{~s}$ and fixed in $2 \%$ paraformaldehyde/1 X PBS again for $10 \mathrm{~min}$. Slides were washed 2 x $5 \mathrm{~min}$ in PBSTB, stained with DAPI in 1 X PBS for $10 \mathrm{~min}$ followed by one wash with PBSTB for $5 \mathrm{~min}$. Slides were mounted in Prolong Gold with DAPI. 
Images were acquired and analyzed as described above. GFP::COSA-1 foci in late pachytene nuclei were counted in at least 5 germ lines/ genotype in the late pachytene.

\section{Data Availability}

Strains and plasmids are available upon request. The authors affirm that all data necessary for confirming the conclusions of the article are present within the article, figures, and tables.

Supplemental Table 1 List of strains generated for this study.

Supplemental Figure 1. ATM-1 limits the accumulation of RAD-51 foci.

Supplemental Figure 2. The impact of atm-1 on noncrossover outcomes is independent of SPO-11 induced breaks.

Supplemental Figure 3. SPO-11-independent COs are induced in atm-1 mutants, revealing carrythrough damage from pre-meiotic events or meiotic $\mathrm{S}$ phase. At the same time, exposure to IR leads to fewer COs in atm-1 mutant background, revealing a defect in converting DSBs to COs in the absence of ATM-1 function.

Supplemental Figure 4. RAD-51 analysis in atl-1 mutants and post-IR exposure.

Supplemental Figure 5. Bioinformatic analysis of potential ATM-1/ATL-1 phosphorylation sites in $C$. elegans DSB proteins.

\section{RESULTS}

\section{ATM-1 helps to promote crossover formation.}

Meiotic DSBs are catalyzed by the conserved topoisomerase SPO11. SPO11 activity is regulated by accessory factors that influence the timing, placement, and extent of DSB formation. ATM influences DSB formation in species as diverged as yeast and mice. In worms, atm-1 mutant animals are homozygous viable and morphologically wild-type, but variably have offspring with reduced viability and fecundity (JONES et al. 2012). In the germ line, an increased number of RAD-51 foci in atm-1 mutant worms has been interpreted to support a widely conserved role in DSB formation (CHECCHI et al. 2014). In C. elegans, mutations in several of the SPO-11 accessory factors, including him-5 and $d s b-2$, lead to a partial impairment in DSB formation, a subsequent decrease in RAD-51 foci, and a lack of COs on a subset of chromosomes (MENEELY et al. 2012; Rosu et al. 2013). Since atm-1 mutants have been shown to exhibit an excess of RAD-51 foci that could reflect an excess of DSBs, we wanted to address how SPO-11 accessory factors and ATM-1 interact to impact CO formation. To test this, we constructed atm-1;him-5 and atm-1;dsb-2 double mutants and examined bivalent formation in single and double mutants by whole mount fixation and DAPI-staining. 
The atm-1; him-5 and atm-1;dsb-2 double mutants contained significantly fewer bivalent chromosomes compared to him-5 and $d s b-2$ single mutants (Figure 1A, 1B, $\mathrm{P}<0.01$ ). The 6 DAPI bodies observed in almost all wild-type germ cells correspond to the 6 bivalents formed between each pair of homologous chromosomes. In him-5 and $d s b-2$ single mutants, the number of DAPI bodies is increased since the non-exchange chromosomes separate from one another into discrete masses, or univalents (Figure 1A, 1B and (MeneELY et al. 2012; Rosu et al. 2013)). The atm-1 single mutant showed $2 \%$ of nuclei with fewer than 6 DAPI bodies (Figure 1A, 1B). A subset of these may reflect whole chromosome fusions as the result of DNA repair defects (JONES et al. 2012). The formation of X:autosome fusions could explain the appearance of heritable, high frequency HIM (high incidence of males) lines in atm-1 mutants (JONES et al. 2012). Surprisingly, close to 10\% of atm-1 mutant nuclei had 7 DAPI bodies. The appearance of these DAPI-bodies was similar to him-5-with five well-formed bivalents and 2 uniformly-sized univalents - which would explain the 2-3\% HIM phenotype in the homozygous stocks. When atm-1 mutations were combined with him-5 or $d s b-2$, the percentage of univalents chromosomes was significantly increased. This was most striking in atm-1; him-5 where $>40 \%$ of diakinesis-stage nuclei contain $>8$ DAPI bodies, the equivalent of two or more chromosomes with defective COs. Thus, while atm-1 mutant animals have been reported to exhibit an increase in RAD-51 foci (CHECCHI et al. 2014), fewer COs appeared to form.

To validate these results, we also quantified COs using GFP::COSA-1, a fusion protein that localizes to the chiasma formed between homologs (YOKOO et al. 2012). Since worm chromosomes usually only receive a single CO, GFP::COSA-1 is seen as a single focus per homolog pair starting in mid-late pachytene. As reported previously, most him-5 mutant nuclei have only 5 GFP::COSA-1 foci (MACHOVINA et al. 2016) reflecting the loss of X chromosome CO formation (Figure 1C). Similarly, $\sim 10 \%$ of nuclei in atm-1 mutants contained only 5 GFP::COSA-1 foci in accord with fraction of diakinesis-stage nuclei that contain 7 DAPI bodies in this mutant (Figure 1C). The number of GFP::COSA-1 foci was also significantly reduced in atm-1; him-5 compared to him-5 (Figure 1C, $\mathrm{P}<0.0001)$. Together these data implicate $a t m-1$ as a pro-CO factor in C. elegans.

We further tested the impact of atm- 1 loss on CO formation by examining recombination rates. We examined recombination rates in two large regions of chromosome III, the intervals between $d p y$ 18, unc- 64 which comprises $>13 \mathrm{cM}$ of the right arm of the chromosome and between $d p y-1$, lon-1 which spans $\sim 14 \mathrm{cM}$ from the middle of the left arm into the middle of the central gene cluster. In both intervals, no significant change in genetic distance was found in atm-1 single mutants compared to 
wild type (Table 1A, 1B). However, atm-1; him-5 mutants gave a lower recombination rate compared to him-5 mutants in both regions of chromosome III (Table 1A, 1B). These data support the interpretation that atm-1 mutations exacerbate the recombination defects caused by lack of him-5 function. Together, these data strongly argue that COs are reduced in atm-1; him-5 double mutants.

\section{ATM-1 limits the number of early repair intermediates}

To understand the nature of the increased RAD-51 foci in atm-1 mutants (CHECCHI et al. 2014) but the decreased numbers of COs in atm-1; him-5 and $a t m-1 ; d s b-2$ mutants, we considered the possibility that ATM-1 might have different roles in otherwise wild-type versus CO-limiting situations. In this scenario, ATM-1 might limit DSBs under normal conditions (leading to the observed increase in RAD51 foci), but when confronted with sub-threshold COs (COs on some chromosomes, but not all), might function in a regulatory feedback loop to retain DSB activity (leading to reduced RAD-51 signals and fewer COs in the mutants). To determine if these different scenarios exist, we quantified RAD-51 foci in atm-1 mutants. We note that prior studies showed a $>95 \%$ concordance between RAD-51 foci and free ends marked by TUNEL staining, so that the former can be used as a surrogate to assess DSB levels (METS AND MEYER 2009). We first validated the results from the prior studies (CHECCHI et al. 2014): using a different source of RAD-51 antibodies (See Materials and Methods), we also observed an increase in RAD-51 foci in atm-1 mutant germ lines compared to wild type $(\mathrm{P}<0.0001$; Chi-square test; Figure 2B-D, S1A and S1B). In atm-1; him-5 and atm-1;dsb-2 double mutants, distribution of RAD-51 foci in the pachytene germ line was altered compared to either single mutants $(\mathrm{p}<0.0001$; Chisquare test; Figure 2D-I). Average RAD-51 levels were also elevated in atm-1; him-5 compared to him5 ( $\mathrm{p}=0.03$; Wilcoxon matched-pairs signed rank test, Figure $2 \mathrm{~F}$ ). These differences are particularly striking in light of our observations that COs were decreased in both atm-1; him-5 and atm-1;dsb-2 (Figure 1). Thus, we conclude that RAD-51 dynamics are affected by loss of atm-1 function in wild type and in mutants where crossovers are limiting.

RAD-51 accumulates on single-stranded, resected DNA ends as a filament that is dismantled upon stable strand exchange. Excessive RAD-51 signal would be seen if additional DSBs were present or if the kinetics of RAD-51 filament formation or turnover were altered. To distinguish between these possibilities, we quantified RAD-51 foci in rad-54 mutant animals in which strand invasion cannot occur and therefore RAD-51 filaments accumulate. As expected, we observed increased RAD-51 foci in atm-1 rad-54 single mutants compared to rad-54 (Table 2 and (CHECCHI et al. 2014)). We also see 
more RAD-51 foci in atm-1,rad-54; him-5 mutants compared to rad-54; him-5 (Table 2). In both wildtype and him-5 mutants, loss of atm-1 resulted in $\sim 5$ additional DSBs.

In addition to SPO-11 mediated DSBs, unrepaired mitotic or meiotic S phase DNA damage can contribute to pachytene accumulation of RAD-51. To determine if such damage is present in atm-1 mutants, we analyzed RAD-51 foci in spo-11 and atm-1; spo-11 in which meiotic DSBs are not formed. If pre-meiotic (or meiotic S-phase) damage were carried through into pachytene, RAD-51 foci should be more prevalent in atm-1;spo-11 compared to spo- 11 . We previously showed that $\sim 10 \%$ of spo-11 nuclei have GFP::COSA-1 foci and elicit CO feedback mechanisms (MACHOVINA et al. 2016). This result was supported here by the appearance of a small number of RAD-51 foci in the pachytene region of spo-11 mutants. By contrast, we saw very few RAD-51 foci in the pre-meiotic and meiotic regions of atm-1;spo-11 (Figure 3A, 3F, S1C and S1D). These results suggest that the extra RAD-51 foci in $\mathrm{atm}-1$ pachytene nuclei are not a consequence of pre-meiotic damage. Instead, these results point to a role for ATM-1 in limiting meiotic DSB formation and/or the early processing of these DSBs.

\section{RAD-51 loading is impaired in atm-1 mutant animals}

We next set out to determine if atm-1 mutants might be defective in RAD-51 filament formation and/or processing. Since the appearance and disappearance of RAD-51 foci is influenced both by the extent of SPO-11 activity and the kinetics of RAD-51 loading and disassembly, it can be difficult to tease out the impact of altered DSB dynamics from altered behavior of RAD-51. To specifically examine the requirement for ATM-1 in RAD-51 loading, we therefore assayed RAD-51 focus formation in backgrounds where meiotic breaks are not made, comparing atm-1;spo-11 with spo-11 at various time points after exposure to IR. Surprisingly, at 1 hour-post IR, we observed many fewer RAD-51 foci in atm-1; spo-11 double mutants compared to spo-11 (Figure 3B, 3G, S1E and S1F). These results intimate that early RAD-51 accumulation is impaired in atm-1 mutants. At 2 hours-post IR, the number of RAD-51 foci in atm-1;spo-11 reached levels comparable to spo-11 1-hour-post IR (Figure 2C, 2H, $\mathrm{S} 1 \mathrm{G}$ and $\mathrm{S} 1 \mathrm{H})$. RAD-51 foci in atm-1;spo-11 and spo-11 decreased comparably as shown by the RAD-51 signals at 4 and 8 hrs post-IR (Figure 2D, 2E, 2I and 2J). Thus, while RAD-51 focus formation may be affected by loss of atm-1, processing/removal of RAD-51 appears to be normal. While IR-induced and SPO-11 induced breaks are not treated identically in cells (MACAISNE et al. 2018), these data raise the possibility that the excess RAD-51 foci seen in atm-1, atm-1; him-5 and atm- 
$1 ; d s b-2$ are not due to impaired RAD-51 processing, but rather most likely result from additional meiotic DSBs.

\section{ATM-1 inhibits homolog-independent repair, channeling DSBs towards inter-homolog recombination}

The bulk of meiotic DSBs are repaired through homologous recombination using either IH-HR or ISHR repair pathways, and among these two pathways, only IH-HR can create chiasmata. We hypothesized that ATM-1 promotes CO formation by inhibiting processes that do not engage the homolog, thus channeling more DSBs going through IH-HR. Without this inhibition, DSBs would preferentially be repaired through IS-HR or as noncrossovers, leading to a deficit in COs. To test this hypothesis, we took advantage of two mutations that are known to impair homolog-independent repair: brc-1 and smc-5 (ADAMO et al. 2008; BICKEL et al. 2010). Mutants carrying either mutation had a small percentage of diakinesis-stage nuclei with chromosome fragments. In syp mutants where synaptonemal complex (SC) formation is impaired, the homolog is not readily available for HR and ISHR is thought to be the major DSB repair pathway (ADAMO et al. 2008; BICKEL et al. 2010; MACAISNE et al. 2018). In the syp mutant background, both brc-1 and smc-5 showed increased chromosome fragmentation. In contrast to brc-1;syp-1 and smc-5;syp-1, we did not observe chromosome fragments in atm-1;syp-1 mutants (Figure 4) indicating that all DSBs are repaired in this mutant context. By contrast, in atm-1; brc-1 mutants, we found more nuclei with fragments compared to brc-1 mutants (Figure 4, $\mathrm{P}<0.05$ ). A similar result was observed in atm-1;smc-5 mutants, in which $\sim 50 \%$ more nuclei contained DNA fragments compared to the $s m c-5$ single mutant. Further, atm-1;smc-5; brc-1 triple mutants revealed a substantially higher percentages of nuclei with fragments compared to $s m c-5 ; b r c-1$ double mutants (Figure 4).

To determine if the increased number of chromosome fragments in the atm-1 mutants arises simply as a result of excess meiotic DSBs formed in this background, we reasoned that atm- 1 should have no impact on the repair of DSBs induced by IR in the smc-5;spo-11(-) background. Consistent with our results in spo-11(+), more nuclei with fragments were observed in atm-1;smc-5;spo-11 mutants compared to smc-5;spo-11 after exposure to 10 Gy IR, while a similar percentage of nuclei with fragments was apparent in non-irradiated smc-5;spo-11 and atm-1;smc-5;spo-11 (Figure S2). Together, these data suggest that ATM-1 functions outside of DSB break formation to promoting homologdependent repair. 


\section{ATM-1 functions on SPO-11 dependent and independent DSBs to promote CO formation when the number of DSBs is limiting.}

The $\mathrm{CO}$ defects in atm-1; him-5 and atm-1;dsb-2 appeared stronger than expected based on the mild CO defect in atm-1 single mutants. This led us to investigate whether atm-1 differentially affects DSB outcomes under low DSB versus high DSB situations. We compared CO outcomes in spo-11 and atm1;spo-11 mutants and after exposure to $2 \mathrm{~Gy}, 10 \mathrm{~Gy}$, and 25 Gy IR (MACHOVINA et al. 2016). Upon exposure to 2 Gy or 10 Gy IR, nuclei of atm-1;spo-11 double mutants exhibited greater numbers of DAPI bodies (fewer bivalents) at diakinesis compared to spo-11 single mutants (Figure 5A, S3). The impact on COs is not specific to the spo-11 mutant background as fewer bivalents were also seen in irradiated $a t m-1 ; d s b-1$ compared to $d s b-1$ mutant animals that are also defective in meiotic DSB formation (STAMPER et al. 2013) (Figure 5A, S3). By contrast, no difference in CO outcomes was observed when atm-1 and atm-1;spo-11 mutants were exposed to $25 \mathrm{~Gy}$, both mostly contained 6 DAPI bodies at diakinesis (Figure 5B). Since 1 Gy IR is expected to give $\sim 2$ meiotic DSBs (MACHOVINA et al. 2016) and to increase linearly with dose, these results suggests that a threshold exists somewhere between 20 and 50 DSBs beyond which atm-1 dysfunction in DSB repair is overcome.

Our analysis of atm-1; him-5 mutants provided support for a threshold of DSBs for ATM-1 regulation. As discussed above, in atm-1; him-5 double mutants, DSBs appeared to be shunted into noncrossover repair pathways. However, the addition of $10 \mathrm{~Gy}$ in this genetic background was sufficient to drive DSBs into the IH-HR pathways, leading to 6 bivalents in most diakinesis nuclei (Figure 5B). The exposure of atm-1; him-5 to 10 Gy IR would induce 20 more DSBs, bringing break levels to near those in atm-1; spo-11 + 25Gy. Together these results support a role for ATM-1 in repair pathway choice when the number of DSBs is under a threshold of $\sim 30-50$ DSBs. When above this threshold, ATM-1 function appears to be bypassed.

\section{ATL-1 contributes to CO formation by influencing accumulation of early break intermediates}

One candidate for subsuming ATM functions is ATR, the other major kinase involved in the DNA damage response. ATR is encoded by atl-1 (ATM-Like) and is an essential gene that is required for mitotic DNA repair ((GARCIA-MUSE AND BOULTON 2005), reviewed in (BUDZOWSKA AND KANAAR 2009)). Loss of atl-1 leads to both macro- and micro-nuclei due to the impairment in DNA damage signaling (Figure S4A). In nuclei that go on to make oocytes, we observed nearly $25 \%$ with only 5 DAPI bodies (Figure 6A), presumably reflecting the formation of chromosome fusions in response to 
DNA damage. Another 5\% of nuclei had more than 6 DAPI bodies (Figure 6A), suggesting that, like atm-1, atl-1 is required for a full complement of $\mathrm{CO}$ exchanges. In $d s b-2$ and him-5 mutants, lack of ATL-1 function also reduced CO numbers (Figure 6A). Thus, we conclude that ATL-1, like ATM-1, contributes to $\mathrm{CO}$ formation when DSBs are limiting.

To determine if ATL-1 directly impacts DSBs/early repair processing, we analyzed RAD-51 dynamics. Two aspects of RAD-51 accumulation distinguished atl-1 mutants from wild type. First, the appearance of RAD-51 signals differed: a subset of atl-1 mutant nuclei exhibited very high RAD-51 signals (Figure 7A-C). Since the RAD-51 signal was so extensive in some nuclei, we quantified these images by binning the nuclei based on number of foci that were observed (Figure 7H-J). The second distinct aspect of atl-1 mutants was the timing of RAD-51 focus formation: in atl-1, RAD-51 foci were observed in most nuclei from the transition zone (TZ) through the pachytene-diplotene border in atl-1 (Figures 7C, H); whereas in wild type, foci only started to accumulate in the $\mathrm{TZ}$ and were seen in most nuclei only at mid-pachytene (Figures 7B, 7H, 2B, S1A).

The differences in RAD-51 accumulation, together with the known role for atl-1 in mitotic cell divisions (ABRAHAM 2001; GARCIA-MUSE AND BOULTON 2005; LAWRENCE et al. 2015), led us to explore whether any of RAD-51 foci could be explained by an increase in carry-through damage from mitotic divisions. In spo-11; atl-1, there was substantial RAD-51 signal in the pachytene region indicating the presence DNA damage that arose independently from meiotic-induced DSBs (Figure 7D; compare with spo-11 in Figure S1C). We note that this damage can contribute to CO formation as we saw an increase in bivalents in spo-11; atl-1 diakinesis-stage oocytes (Figure 6B). Irradiation of spo-11; atl-1 mutants with 10 Gy IR showed efficient RAD-51 loading within 1 hour post-IR and, as in the atl-1 background, a subset of the signal appeared in stretches (as opposed to smaller foci; Figure S4D). Since these stretches were not seen in the irradiated spo-11 mutant germ lines (Figure S1E, G), we infer that these stretches do not result from two adjacent DSBs, but rather from a defect that is specific to the atl-1 mutant. Similar stretches of RAD-51 have been seen in meiotic mutants with defects in RAD-51 filament maturation (METS AND MEYER 2009; WARD et al. 2010), raising the possibility that ATL-1 limits/antagonizes RAD-51 loading. atl-1 loss would therefore lead to excessive and perhaps, untimely, RAD-51 filament formation.

We also observed RAD-51 foci in the TZ of atl-1, him-5 double mutant animals (Figure 7A, E). In this mutant background, the kinetics of RAD-51 formation and loss are similar to atl-1 single mutants, 
appearing earlier and brighter that in wild type (Figures 7H). However, the number of nuclei with RAD-51 staining was reduced in atl-1,him-5 compared to atl-1 mutants, as expected since him-5 mutations partially impair DSB formation. The number of RAD-51-positive nuclei was also reduced compared to him-5 (Figure 7E-F, 7H-I): whereas upwards of 70\% of him-5 mutant nuclei stained weakly for RAD-51 at its peak, fewer than 30\% of atl-1, him-5 nuclei showed RAD-51 foci (Figure $7 \mathrm{H}-\mathrm{I})$. Of those that had foci, a subset had the very strong RAD-51 signals that were associated with carry-through damage, described above. Thus, $30 \%$ is an overrepresentation of the number of nuclei with bona fide meiotic damage. These data show that atl-1 and him-5 both contribute to RAD-51-focus formation and that atl-1 is epistatic to him-5 for timing of RAD-51 formation.

In yeast and mice, ATM and ATR function together to establish DSB homeostasis (CARBALLO et al. 2013): ATM inhibiting DSBs to prevent over-accumulation; ATR promoting DSBs to ensure sufficient COs can be made. We therefore wanted to know what the impact on meiotic $\mathrm{CO}$ formation would be when both atm-1 and atl-1 are mutated. To our surprise, atm-1; atl-1 mutants showed a severe defect in CO formation: whereas both atm-1 and atl-1 single mutants exhibited mild CO defects (Figure 6A and 6C), in the double mutants, $>70 \%$ of nuclei had CO defects, of which $>50 \%$ contain one or more pairs of univalents. Loss of both atm-1 and atl-1 also exacerbated the CO defect associated with him-5 and $d s b-2$ (Figure 6C). Thus, despite seemingly antagonistic roles on RAD-51 formation, atm-1;atl-1 double mutants are significantly impaired in $\mathrm{CO}$ formation.

Consistent with these results, we saw very few RAD-51 foci in early and mid-pachytene nuclei in $\mathrm{atm}$ 1; atl-1 (Figures 7G-H, J). At late pachytene, almost all nuclei stained strongly for RAD-51, a phenotype seen in neither of the single mutants. It is unlikely that all of these nuclei are destined for apoptosis since we observed diakinesis-stage oocytes with well-formed bivalents (Figure 6C). Instead this data suggests a change in CO regulation in the double mutant (discussed below).

\section{CO feedback is impacted by loss of atm-1 and atl-1}

Defects in CO formation are thought to activate surveillance systems that feed back onto the break machinery to maintain DSB competency when COs are not detected (ROSU et al. 2013; STAMPER et al. 2013; MACHOVINA et al. 2016; NADARAJAN et al. 2017; PATTABIRAMAN et al. 2017). The activity of this feedback mechanism is spatially observed in the pachytene germ line as an extended region of DSB-1 and DSB-2 staining in CO-deficient worms (ROSU et al. 2013; STAMPER et al. 2013). We reasoned that the increased number of RAD-51 foci observed in atm-1 mutants might be explained by 
changes in DSB-2 regulation. We observed that DSB-2 staining in atm-1 mutants was shifted proximally - turning on slightly later than in wild type relative to the onset of leptotene (Figure 8). It also persisted slightly longer, taking on average $\sim 7 \%$ more of the pachytene region than wild type (Figure 8). The small number of excess DSBs in atm-1 may be attributed to this increased window of opportunity for DSB-2- (and by inference, DSB-1-) dependent breaks. We also observed that atm-1 activity was not required to induce (or maintain) the extended domain of DSB-2 in him-5 mutants (Figure 8). Thus, we posit that the reduction in COs in atm-1; him-5 cannot be explained by inhibition of DSB-2.

In atl-1 mutants, DSB-2 staining spanned only $\sim 33 \%$ of the leptotene to pachytene region whereas it comprised $\sim 45 \%$ in wild type (Figure 8 ). This result suggests that the CO-dependent deactivation of DSB-2 occurred more rapidly in atl-1 mutants. him-5 is epistatic to atl-1, as was seen by the slight delay in DSB-2 onset and much extended domain of staining in atl-1, him-5 double mutants. These phenotypes are best explained by HIM-5's role in promoting DSB formation: delaying the formation of DSBs and preventing DSBs on the X chromosome (MENEELY et al. 2012).

In the atm-1; atl-1 mutants, the DSB-2 region was distinct from either single mutant: DSB-2 was activated as in wild type, yet it persisted until very late pachytene. The extension of DSB-2 staining was similar to that seen in him-5 mutants, suggesting it may also reflect the diminished COs that form in the double mutants.

\section{DISCUSSION}

ATM-1 and ATR-1 have both unique and overlapping functions in worm meiosis that influence the formation of COs. We have shown that these genes have antagonistic and synergistic roles in DSB and $\mathrm{CO}$ formation which is summarize in our proposed model in Figure 9. We posit that upon SPO-11 activation, a small number of initial DSBs are made $(<10)$ that are sufficient to activate ATM-1 and ATL-1. Both proteins would then influence the activation of DSB-2 (perhaps through DSB-1): ATL-1 directly and ATM-1 through the regulation of early events post-DSB formation, perhaps resection. The lack of input from ATL-1 would explain the reduction in DSBs seen in atl-1 mutants. Delayed resection could explain the delayed activation of the DSB-2 feedback loop and its prolonged localization in atm-1 mutants. We propose that ATM-1 and ATL-1 then influence the transition from resection to an IH-CO competent RAD-51 filament. In the case of ATM-1, our atm-1;spo-11+IR data 
predict a role in the timely recruitment of RAD-51. This delay could be a consequence of its role in resection or its role in influencing RAD-51 loading. Further studies will be required to determine if ATM-1 has the same impact on RAD-51 recruitment at SPO-11-induced breaks.

To our surprise, we found that despite increased RAD-51 foci, COs are diminished in atm-1 mutants. This reduction could due to the ability of ATM-1 to influence the timely recruitment of RAD-51 (assuming SPO-11 induced breaks are treated the same at IR-induced breaks) and/or to shunt meiotic DSBs into the IH-CO repair pathway. In its absence, noncrossover repair pathways are favored. Thus, atm-1 appears to function antagonistically on different aspects of $\mathrm{CO}$ formation, limiting the total number of DSBs, but increasing the likelihood that DSBs are repaired by IH-CO repair. ATL-1 also appears to have an antagonistic function with decreased numbers of DSBs yet excessive RAD-51 loading seen in the atl-1 mutant animals. These mutually antagonistic behaviors can be explained by negative and positive feedback loops and built-in functional redundancy (Figure 9) that illustrate the extensive and precise machinations required to ensure that COs occur on each chromosome.

Our model posits that, as in other systems (reviewed in (MORIEL-CARRETERO et al. 2018)), DSBs activate ATM-1 to promote timely resection and subsequent activation of ATL-1. Resected ends and ATL-1 would both promote a secondary wave of DSBs that are induced through activation of DSB-2, and by inference DSB-1 (STAMPER et al. 2013). This explains the decreased RAD-51 foci in atl-1 mutants and the delay in DSB-2 loading in atm-1 mutants. The increased number of RAD-51 foci in atm-1 mutants might suggest that ATM-1 negatively regulates DSB formation. Alternatively, the 5-6 extra DSBs (Table 2) that are made could also be explained by persistent activation of the CO surveillance system ((MACHOVINA et al. 2016), Figure 9). In this case, a bias for IS-HR or noncrossovers in atm-1 mutants would result in later CO formation and tardy deactivation of DSB-1/2. ATL-1 (and perhaps ATM-1) are likely to be regulating DSBs and DSB-2 localization through phosphorylation of SPO-11 and/or one or more its accessory factors. Bioinformatic analysis identified potential (S/T)Q sites in all of the ten known factors that influence DSB formation supporting the possibility that one or more are direct targets of ATL-1 (or ATM-1) (Fig S5). Our analyses of atm1; dsb-2 and atm-1; him-5 double mutants showed increased DSBs (seen as RAD-51 foci), ruling out DSB-2 and HIM-5 as the sole targets of ATM/ATR signaling, although they may have redundant functions. Further studies are needed to identify the relevant targets in the DSB machinery. 
Our observation that RAD-51 loading is delayed in atm-1 mutants post-IR suggests that ATM-1 facilitates efficient formation of the RAD-51 filament. In mitosis, both ATM and ATR activate resection activities with ATR functioning as well to attenuate Exo1-mediated activities (reviewed in (GOBBINI et al. 2013)). In meiosis, recent studies have shown that yeast ATM1/Tel1 promotes resection of early DSBs (JOSHI et al. 2015) and that mouse ATM helps to initiate and promote resection (MIMITOU et al. 2017). A defect in resection could explain the delay in RAD-51 recruitment in atm-1;spo-11 post-IR. A likely downstream target to regulate resection and RAD-51 loading (serving as protein X in Figure 9) is RAD-50, whose homologs are targets of ATM signaling in other systems (GATEI et al. 2011). In worms, RAD-51 loading in the early-mid pachytene region requires RAD-50 activity, whereas late pachytene loading is RAD-50-independent (HAYASHI et al. 2007). The transition between these two states is thought to correspond to the switch from IH-HR to IS-HR. By promoting RAD-50 activity, ATM-1 could therefore assure the repair of meiotic DSBs by IH-HR; in its absence, RAD-50 activity would be attenuated and IS-HR/ noncrossover repair would be favored.

ATR has also been implicated in resection control, both promoting and restraining extensive EXO1 activity (TOMIMATSU et al. 2017). Our observation that a subset of ATR mutants present with large RAD-51 aggregates intimates that the latter function, at least, of ATR may be conserved in worm ATL-1. The rapid disappearance of nuclear localized DSB-2 in atl-1 mutants could be explained by the formation of longer resected in ends that would more rapidly be converted into IH-COs, leading to cessation of DSB formation by CO feedback (MACHOVINA et al. 2016). Together with our analysis of ATM-1, these data raise the intriguing possibility that different resection tract lengths could influence the crossover vs noncrossover decision.

Based on their mutually antagonistic behavior on DSBs/RAD-51 foci formation, we explored the impact of loss of both ATM-1 and ATL-1 functions. In atm-1; atl-1 double mutants, both RAD-51 foci and COs are decreased (Figure S4D and 6C). This suggests that atl-1 has an additional role in promoting RAD-51 loading that is redundant with ATM-1. Although depicted as a shared target gene, this may reflect one or more downstream roles. We envision that in the double mutant background, the DSB feedback loop is activated via resected ends (through DSB-1 or other accessory factors), the ends are hyper-resected due to loss of atl-1, but RAD-51 loading is significantly delayed due to the joint functions of ATL-1 and ATM-1 on RAD-51 loading which are relieved when the block to IS-HR is relaxed in late pachytene, leading to the excessive loading of RAD-51 in the atm-1;atl-1 double mutant. 
These data reveal the complex interplay between ATM and ATR signaling in meiosis that ultimately helps determine both the number of DSBs and the formation of IH-COs. These studies highlight multiple potential targets of ATM-1 and ATL-1 and provide the basis for the future identification and analysis of specific substrates. These studies also illuminate the evolutionary conserved of antagonism between ATM and ATR that is necessitated by the requirement for CO formation on each chromosome.

\section{REFERENCES}

Abraham, R. T., 2001 Cell cycle checkpoint signaling through the ATM and ATR kinases. Genes Dev 15: 2177-2196.

Adamo, A., P. Montemauri, N. Silva, J. D. Ward, S. J. Boulton et al., 2008 BRC-1 acts in the intersister pathway of meiotic double-strand break repair. EMBO Rep 9: 287-292.

Anderson, C. M., A. Oke, P. Yam, T. Zhuge and J. C. Fung, 2015 Reduced Crossover Interference and Increased ZMM-Independent Recombination in the Absence of Tel1/ATM. PLoS Genet 11: e1005478.

Barlow, C., S. Hirotsune, R. Paylor, M. Liyanage, M. Eckhaus et al., 1996 Atm-deficient mice: a paradigm of ataxia telangiectasia. Cell 86: 159-171.

Barlow, C., M. Liyanage, P. B. Moens, M. Tarsounas, K. Nagashima et al., 1998 Atm deficiency results in severe meiotic disruption as early as leptonema of prophase I. Development 125: 4007-4017.

Bickel, J. S., L. Chen, J. Hayward, S. L. Yeap, A. E. Alkers et al., 2010 Structural maintenance of chromosomes (SMC) proteins promote homolog-independent recombination repair in meiosis crucial for germ cell genomic stability. PLoS Genet 6: e1001028.

Brenner, S., 1974 The genetics of Caenorhabditis elegans. Genetics 77: 71-94.

Budzowska, M., and R. Kanaar, 2009 Mechanisms of dealing with DNA damage-induced replication problems. Cell Biochem Biophys 53: 17-31.

Carballo, J. A., S. Panizza, M. E. Serrentino, A. L. Johnson, M. Geymonat et al., 2013 Budding yeast ATM/ATR control meiotic double-strand break (DSB) levels by down-regulating Rec114, an essential component of the DSB-machinery. PLoS Genet 9: e1003545.

Checchi, P. M., K. S. Lawrence, M. V. Van, B. J. Larson and J. Engebrecht, 2014 Pseudosynapsis and decreased stringency of meiotic repair pathway choice on the hemizygous sex chromosome of Caenorhabditis elegans males. Genetics 197: 543-560.

Cooper, T. J., K. Wardell, V. Garcia and M. J. Neale, 2014 Homeostatic regulation of meiotic DSB formation by ATM/ATR. Exp Cell Res 329: 124-131.

Garcia, V., S. Gray, R. M. Allison, T. J. Cooper and M. J. Neale, 2015 Tel1(ATM)-mediated interference suppresses clustered meiotic double-strand-break formation. Nature 520: 114-118.

Garcia-Muse, T., and S. J. Boulton, 2005 Distinct modes of ATR activation after replication stress and DNA double-strand breaks in Caenorhabditis elegans. EMBO J 24: 4345-4355.

Gatei, M., B. Jakob, P. Chen, A. W. Kijas, O. J. Becherel et al., 2011 ATM protein-dependent phosphorylation of Rad50 protein regulates DNA repair and cell cycle control. J Biol Chem 286: 31542-31556. 
Gobbini, E., D. Cesena, A. Galbiati, A. Lockhart and M. P. Longhese, 2013 Interplays between ATM/Tel1 and ATR/Mec1 in sensing and signaling DNA double-strand breaks. DNA Repair (Amst) 12: 791-799.

Gray, S., R. M. Allison, V. Garcia, A. S. Goldman and M. J. Neale, 2013 Positive regulation of meiotic DNA double-strand break formation by activation of the DNA damage checkpoint kinase Mec1(ATR). Open Biol 3: 130019.

Hayashi, M., G. M. Chin and A. M. Villeneuve, 2007 C. elegans germ cells switch between distinct modes of double-strand break repair during meiotic prophase progression. PLoS Genet 3: e191.

Jones, M. R., J. C. Huang, S. Y. Chua, D. L. Baillie and A. M. Rose, 2012 The atm-1 gene is required for genome stability in Caenorhabditis elegans. Mol Genet Genomics 287: 325-335.

Joshi, N., M. S. Brown, D. K. Bishop and G. V. Borner, 2015 Gradual implementation of the meiotic recombination program via checkpoint pathways controlled by global DSB levels. Mol Cell 57: 797-811.

Joyce, E. F., M. Pedersen, S. Tiong, S. K. White-Brown, A. Paul et al., 2011 Drosophila ATM and ATR have distinct activities in the regulation of meiotic DNA damage and repair. J Cell Biol 195: 359-367.

Lange, J., J. Pan, F. Cole, M. P. Thelen, M. Jasin et al., 2011 ATM controls meiotic double-strandbreak formation. Nature 479: 237-240.

Lawrence, K. S., T. Chau and J. Engebrecht, 2015 DNA damage response and spindle assembly checkpoint function throughout the cell cycle to ensure genomic integrity. PLoS Genet 11: e1005150.

Macaisne, N., Z. Kessler and J. L. Yanowitz, 2018 Meiotic Double-Strand Break Proteins Influence Repair Pathway Utilization. Genetics 210: 843-856.

Machovina, T. S., R. Mainpal, A. Daryabeigi, O. McGovern, D. Paouneskou et al., 2016 A Surveillance System Ensures Crossover Formation in C. elegans. Curr Biol 26: 2873-2884.

MacQueen, A. J., and A. Hochwagen, 2011 Checkpoint mechanisms: the puppet masters of meiotic prophase. Trends Cell Biol 21: 393-400.

Martini, E., R. L. Diaz, N. Hunter and S. Keeney, 2006 Crossover homeostasis in yeast meiosis. Cell 126: 285-295.

McClendon, T. B., R. Mainpal, F. R. Amrit, M. W. Krause, A. Ghazi et al., 2016 X Chromosome Crossover Formation and Genome Stability in Caenorhabditis elegans Are Independently Regulated by xnd-1. G3 (Bethesda) 6: 3913-3925.

Meneely, P. M., O. L. McGovern, F. I. Heinis and J. L. Yanowitz, 2012 Crossover distribution and frequency are regulated by him-5 in Caenorhabditis elegans. Genetics 190: 1251-1266.

Mets, D. G., and B. J. Meyer, 2009 Condensins regulate meiotic DNA break distribution, thus crossover frequency, by controlling chromosome structure. Cell 139: 73-86.

Mimitou, E. P., S. Yamada and S. Keeney, 2017 A global view of meiotic double-strand break end resection. Science 355: 40-45.

Moriel-Carretero, M., P. Pasero and B. Pardo, 2018 DDR Inc., one business, two associates. Curr Genet.

Nadarajan, S., T. J. Lambert, E. Altendorfer, J. Gao, M. D. Blower et al., 2017 Polo-like kinasedependent phosphorylation of the synaptonemal complex protein SYP-4 regulates doublestrand break formation through a negative feedback loop. Elife 6.

Pattabiraman, D., B. Roelens, A. Woglar and A. M. Villeneuve, 2017 Meiotic recombination modulates the structure and dynamics of the synaptonemal complex during C. elegans meiosis. PLoS Genet 13: e1006670.

Rosu, S., K. A. Zawadzki, E. L. Stamper, D. E. Libuda, A. L. Reese et al., 2013 The C. elegans DSB-2 protein reveals a regulatory network that controls competence for meiotic DSB formation and promotes crossover assurance. PLoS Genet 9: e1003674. 
Stamper, E. L., S. E. Rodenbusch, S. Rosu, J. Ahringer, A. M. Villeneuve et al., 2013 Identification of DSB-1, a protein required for initiation of meiotic recombination in Caenorhabditis elegans, illuminates a crossover assurance checkpoint. PLoS Genet 9: e1003679.

Tomimatsu, N., B. Mukherjee, J. L. Harris, F. L. Boffo, M. C. Hardebeck et al., 2017 DNA-damageinduced degradation of EXO1 exonuclease limits DNA end resection to ensure accurate DNA repair. J Biol Chem 292: 10779-10790.

Ward, J. D., D. M. Muzzini, M. I. Petalcorin, E. Martinez-Perez, J. S. Martin et al., 2010 Overlapping mechanisms promote postsynaptic RAD-51 filament disassembly during meiotic double-strand break repair. Mol Cell 37: 259-272.

Xu, Y., T. Ashley, E. E. Brainerd, R. T. Bronson, M. S. Meyn et al., 1996 Targeted disruption of ATM leads to growth retardation, chromosomal fragmentation during meiosis, immune defects, and thymic lymphoma. Genes Dev 10: 2411-2422.

Yokoo, R., K. A. Zawadzki, K. Nabeshima, M. Drake, S. Arur et al., 2012 COSA-1 reveals robust homeostasis and separable licensing and reinforcement steps governing meiotic crossovers. Cell 149: 75-87.

Zhang, L., K. P. Kim, N. E. Kleckner and A. Storlazzi, 2011 Meiotic double-strand breaks occur once per pair of (sister) chromatids and, via Mec1/ATR and Tel1/ATM, once per quartet of chromatids. Proc Natl Acad Sci U S A 108: 20036-20041.

\section{ACKNOWLEDGMENTS}

The authors wish to thank Dr. Kara Bernstein, Dr. Arjumand Ghazi, Dr. Arthur Levine, Dr. Nicolas Macaisne, Dr. Brooke McClendon, and Logan Russell for critical reading of the manuscript. Gratitude is extended to Dr. Sarit Smolikove for sharing her anti-RAD-51 antibody. Some strains were provided by the CGC, which is funded by NIH Office of Research Infrastructure Programs (P40 OD010440).

W.L. was funded by Tsinghua University School of Medicine; J.L.Y. was funded by Pennsylvania Formula Funds and NIGMS/NIH (GM104007). 
bioRxiv preprint doi: https//doi org/10.1101/604827 t this version posted April 10,2019 . The copyright holder for this preprint (which was not certified by peer review) is the author/funder, who has granted bioRxiv a license to display the preprint in perpetuity. It is made available under aCC-BY-NC 4.0 International license.

TABLE 1. ATM-1 MUTANTS EXACERBATE DEFECTS IN RECOMBINATION

\begin{tabular}{|c|c|c|c|c|}
\hline Genotype & $\mathbf{N 2}$ & atm-1 & him-5 & atm-1;him-5 \\
\hline \multicolumn{5}{|c|}{$d p y-18$ (III: 8.85), unc-64 (III: 21.20) } \\
\hline $\begin{array}{l}\text { Recombination } \\
\text { rate }\end{array}$ & $12.01 \%(1896)$ & $11.97 \%(2816)$ & $14.22 \%, \#(2475)$ & $10.93 \% * * *(1587)$ \\
\hline \multicolumn{5}{|c|}{$d p y-1$ (III: -15.66), Ion-1 (III: -1.63) } \\
\hline $\begin{array}{l}\text { Recombination } \\
\text { rate }\end{array}$ & $17.56 \%(3995)$ & $15.99 \%(1958)$ & $17.14 \% * * *(2705)$ & $13.78 \% \%^{* * *}(1652)$ \\
\hline
\end{tabular}

TABLE 2. EARLY REPAIR INTERMEDIATES ACCUMULATE IN ATM-1 MUTANTS

\begin{tabular}{ll}
\hline genotype & No. of RAD-51 foci/nucleus \\
\hline rad-54 & $31.08 \pm 1.88$ \\
atm-1;rad-54 & $35.91 \pm 1.64^{\S}$ \\
& \\
rad-54;him-5 & $22.83 \pm 2.35$ \\
atm-1;rad-54;him-5 & $28.82 \pm 3.73^{\dagger}$
\end{tabular}

Analysis of late pachytene nuclei

st test, $p<0.0001$

tt test, $p<0.0001$ 


\section{FIGURE 1}

A

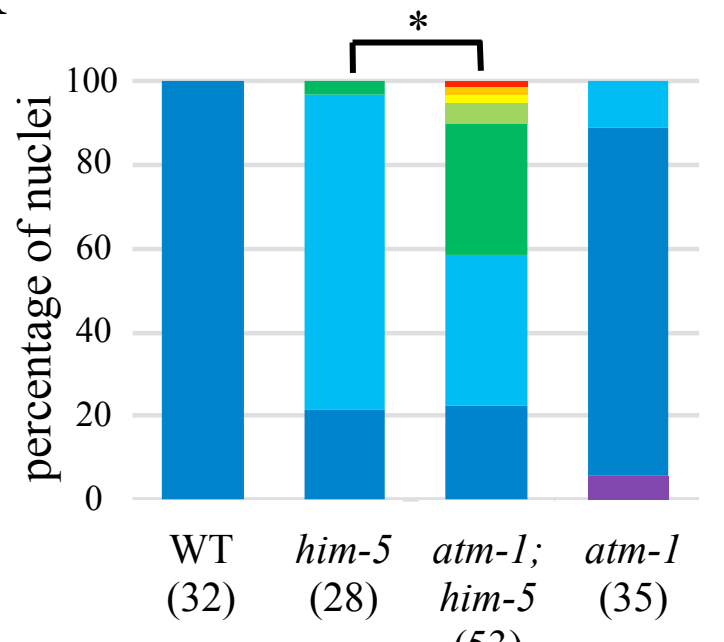

(53)

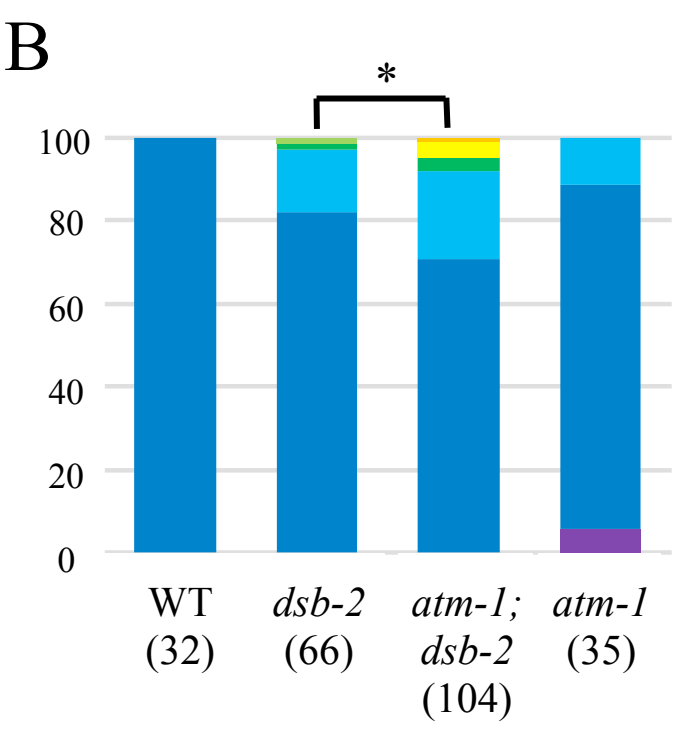

$11 \square 12$

$\mathrm{C}$

\# DAPI bodies

\section{$15 \square 67 \square 8$}
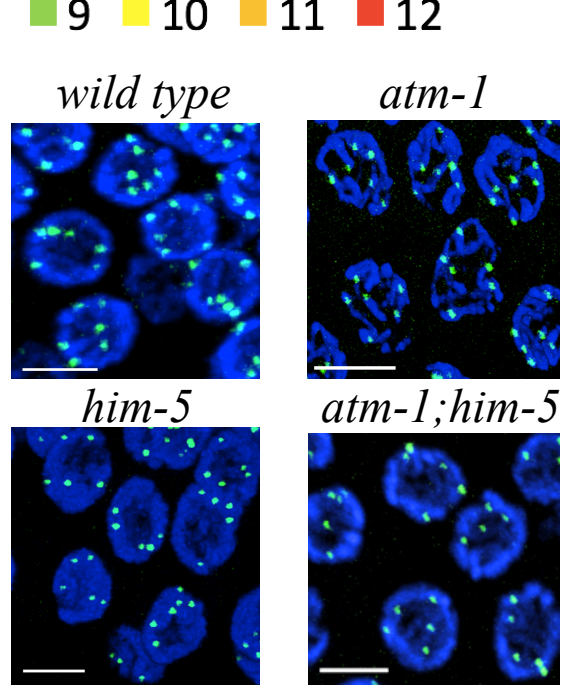

DAPI GFP::COSA-1

Figure 1. atm-1 mutations exacerbate CO defects of DSB-defective mutants (A) him-5 (B) dsb-2. Quantification of DAPI bodies in -1 nuclei. *P $<0.05$, two-tailed Mann Whitney test. (C) Proportion of nuclei with indicated numbers of GFP::COSA-1 foci $* * * * \mathrm{P}<0.0001$, two-tailed Mann Whitney test. Three gonads were analyzed for each genotype. Numbers below each genotype indicate the numbers of nuclei analyzed. (D) Images of him-5 and atm-1; him-5 late pachytene nuclei are shown with DAPI (blue) and GFP::COSA-1 (green). Scale bar $=5 \mu \mathrm{m}$. 


\section{FIGURE 2}

A
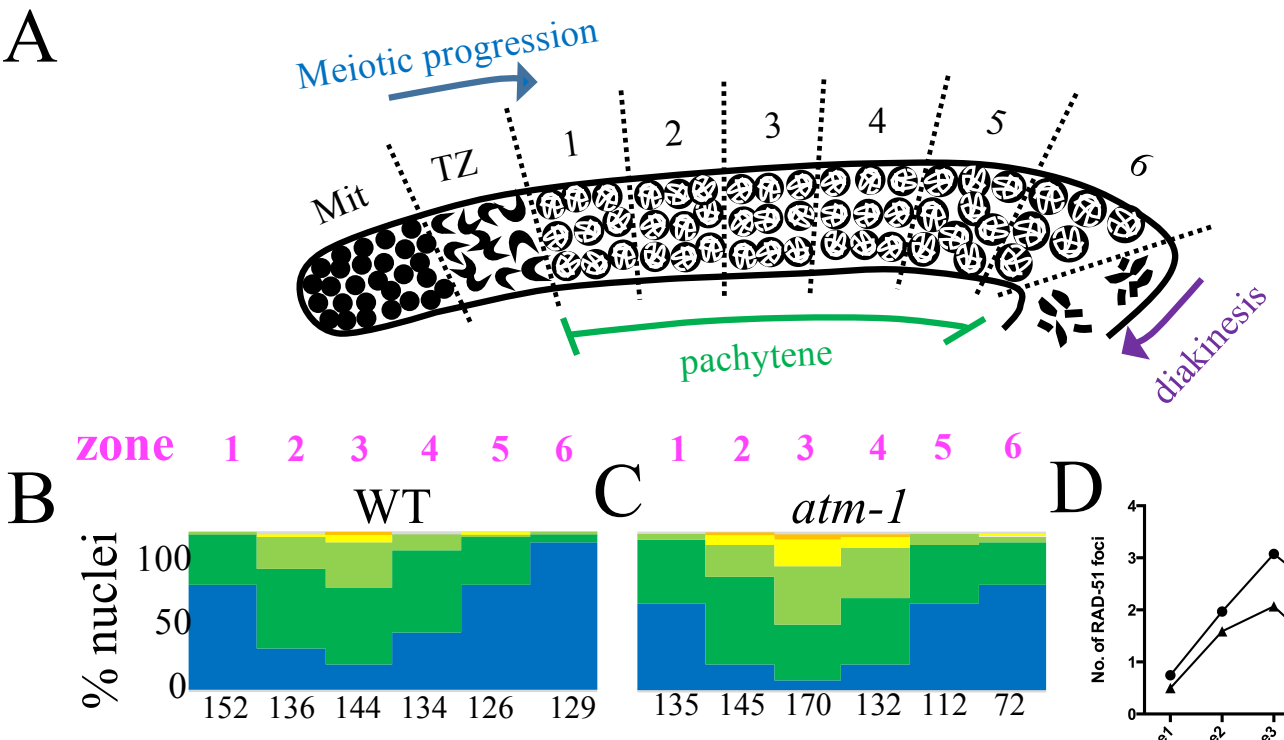

$\mathrm{E}$

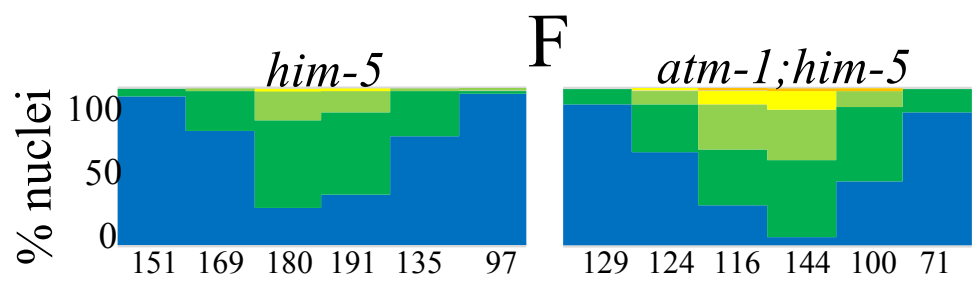

$\mathrm{H}$
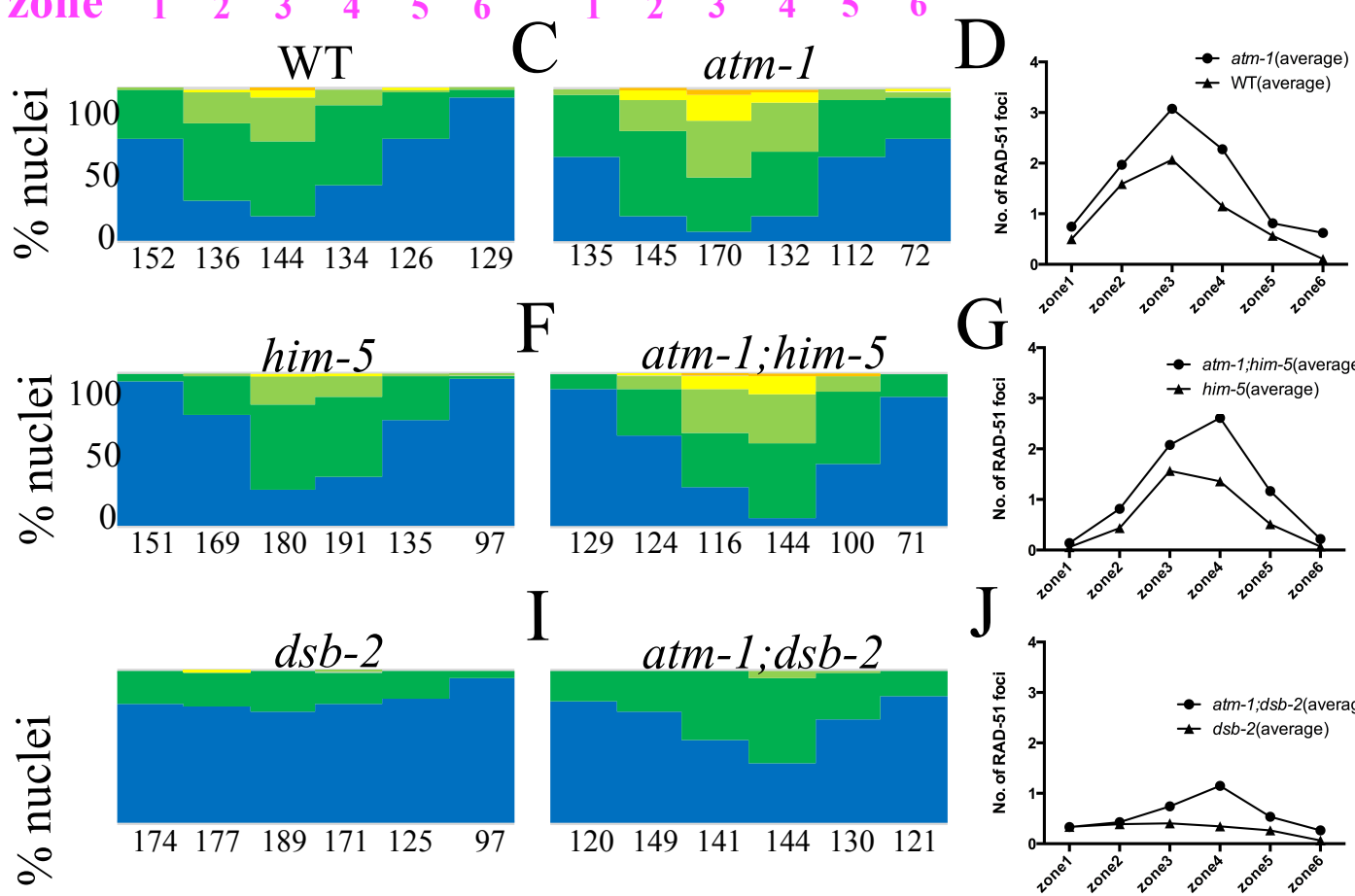

$\mathrm{G}$

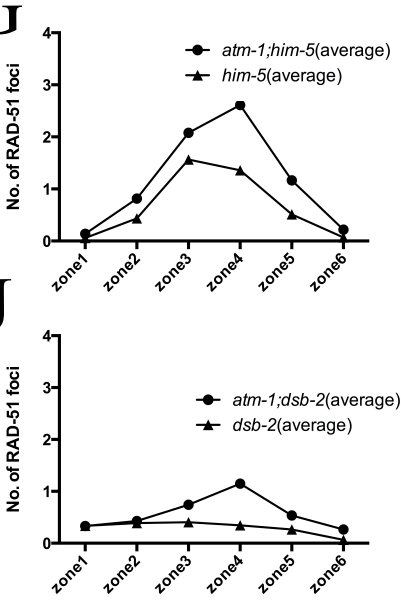

No. of RAD-51 foci $\square 0 \square 1 \sim 2 \square 3 \sim 4 \square 5 \sim 6 \square 7 \sim 8 \square>=9$

Figure 2. Early repair intermediates accumulate to a greater extent in atm-1 mutants. (A) Schematic of the $C$. elegans germline showing regions in which RAD-51 foci were quantified. Mit: Mitotic zone. TZ: transition zone. (B, C, E, F, H, I) The percentage of nuclei in each zone containing the indicated number of RAD-51 foci shown in the color key at the bottom. Numbers represent total \# of nuclei counted/zone for three gonads/genotype. (D, G, J) Comparison of the average number of RAD-51 foci for each genotype.. \# = nuclei scored/ 3 germ lines/ genotype. $\chi^{\text {square }}$, atm-1 vs. N2; atm-1; him-5 vs. him-5; and atm-1;dsb-2 vs. $d s b-2: \mathrm{p}<0.0001$ for each. Representative images of germ lines are shown in Supplemental Figure S1. 


\section{FIGURE 3}

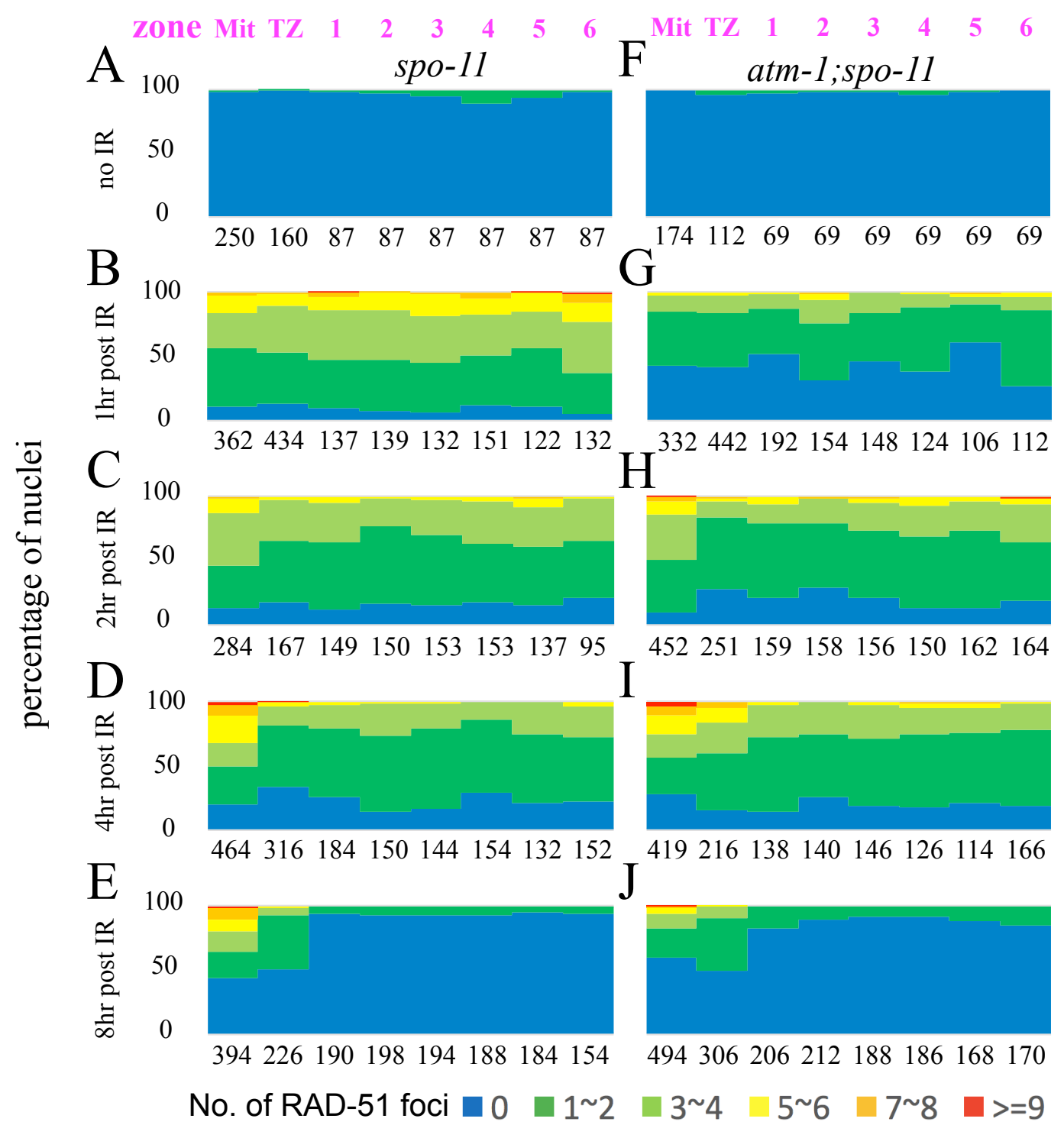

Figure 3. Loading of RAD-51 is delayed in atm-1 mutants. For each region, the percentage of nuclei with a given number of RAD-51 foci are shown as a heat map from $0->9$ as indicated below. Zones are shown in Figure 2A. \# = nuclei scored/ 3 germ lines/ genotype. (A-E) spo-11 mutants. (F-J) atm1; spo-11 mutants. Shown in a time-course post-exposure to $10 \mathrm{~Gy}$ IR (A, F): unirradiated controls (B, $\mathrm{G})$ : 1 hour post-exposure shows reduced loading in atm-1;spo-11. (C, H): 2 hours and (D, I): 4 hours post-exposure loading is almost indistinguishable between control and atm-1;spo-11. (E, J): 8 hours post-exposure, most RAD-51 foci have been removed in the meiotic region of the germ line. We note that repair in the mitotic region is resolved with distinct kinetics. 


\section{FIGURE 4}

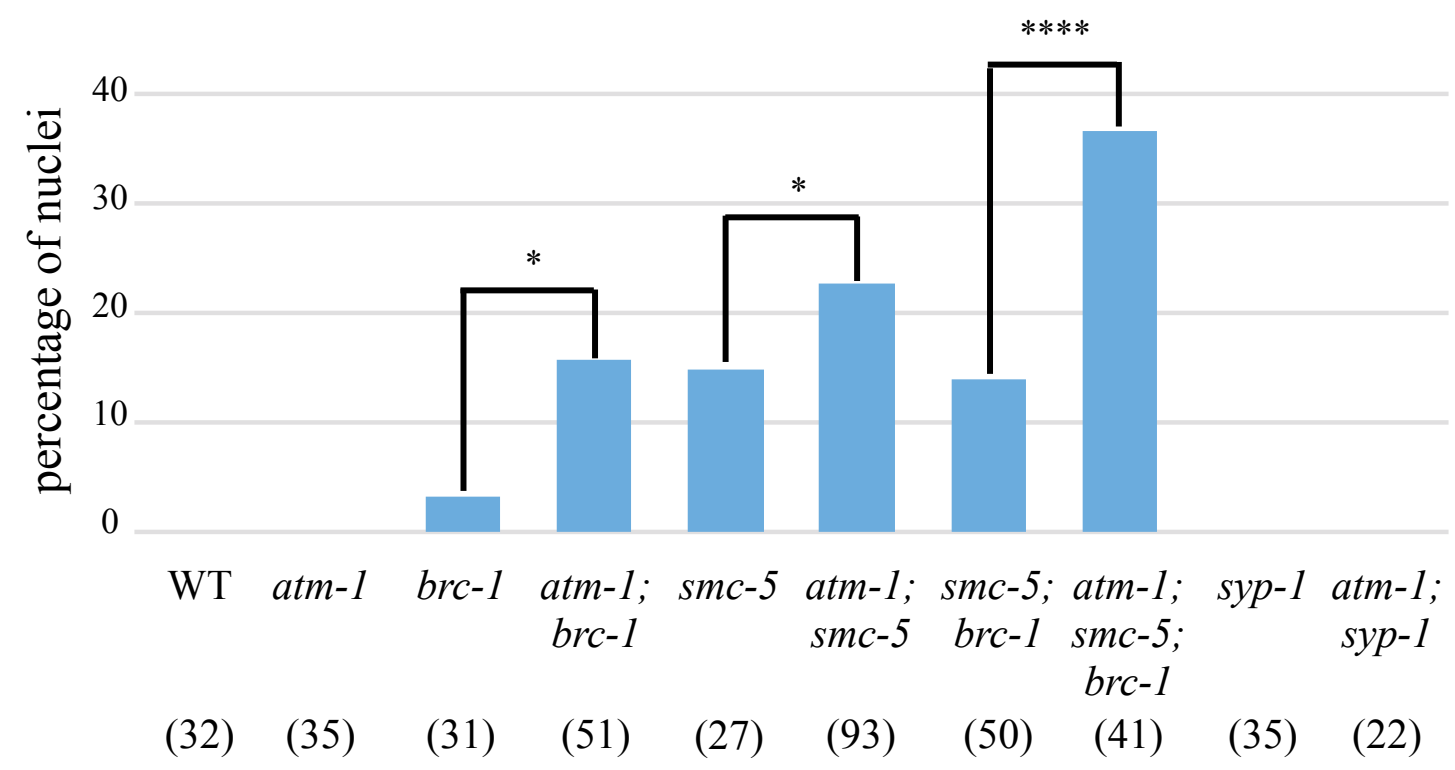

Figure 4. atm-1 mutation increases fragmentation when inter-sister repair is impaired. Percentage of -1 nuclei containing DNA fragments as seen by DAPI staining. Numbers in brackets are the number of nuclei analyzed/ genotype. ${ }^{*} \mathrm{P}<0.05, * * * * \mathrm{P}<0.0001\left(\chi^{2}\right.$ test $)$. 


\section{FIGURE 5}

A

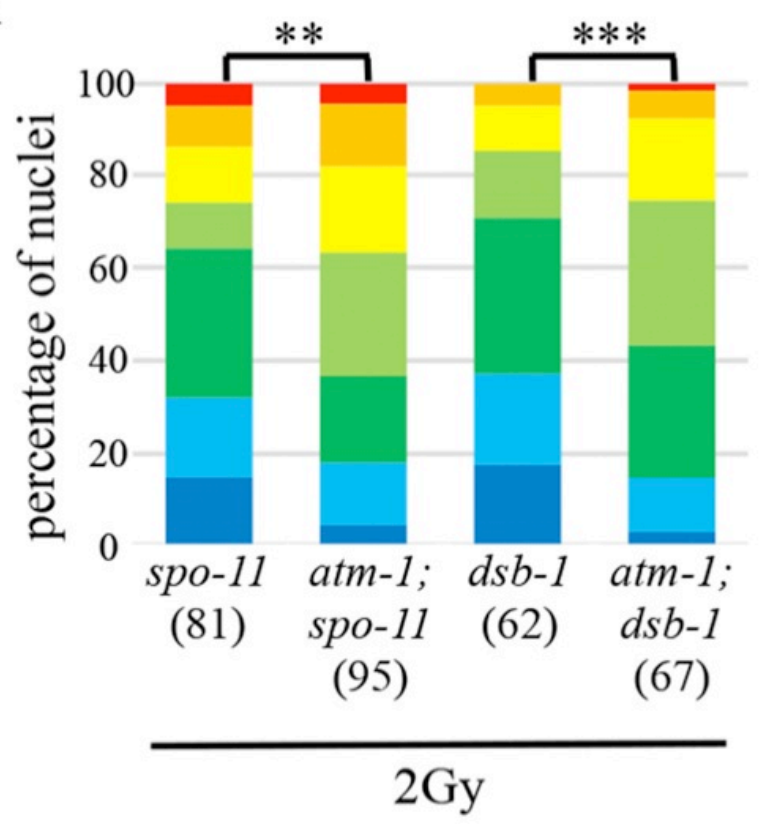

No. of DAPI bodies
B

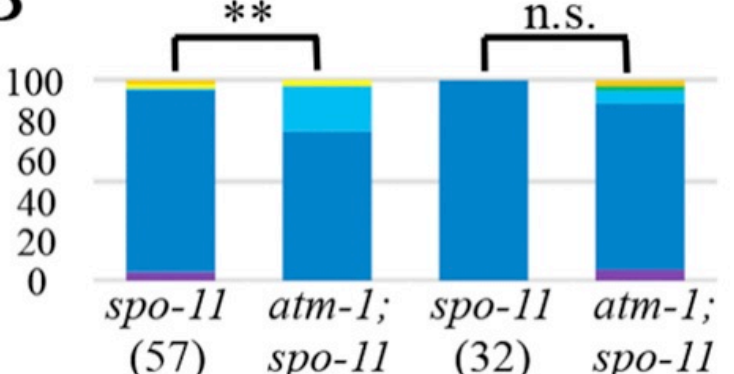

(31)

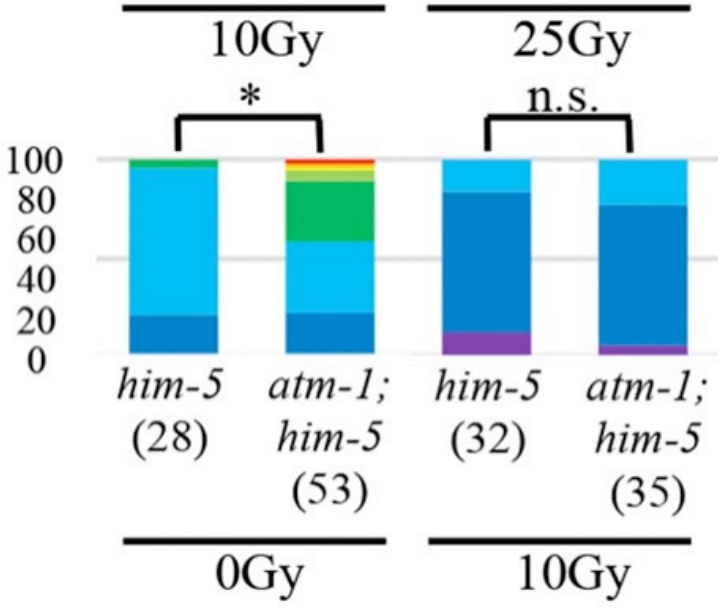

Figure 5. atm-1 acts on exogenous DSBs as well as SPO-11 induced DSBS to promote crossovers within a threshold of total DSBs. Quantification of DAPI bodies in -1 nuclei for different doses of IR. Color key below. (A) 2 Gy IR leads to more COs in atm-1;spo-11 and atm-1;dsb-1 compared to control spo-11 $d s b-1$, respectively. (B, C) atm-1 loss is overpowered by high numbers of DSBs, between 10 Gy and 25 Gy (20-50 DSBs) in atm-1; spo-11 (top); below 10 Gy (20 additional breaks) in atm-1; him-5 ( $\sim 28$ endogenous breaks). ${ }^{*} \mathrm{P}<0.05,{ }^{* *} \mathrm{P}<0.01, * * * \mathrm{P}<0.001$, n.s. $=$ no significant difference, two-tailed Mann Whitney test. 


\section{FIGURE 6}

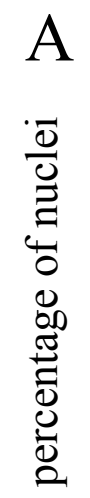

0
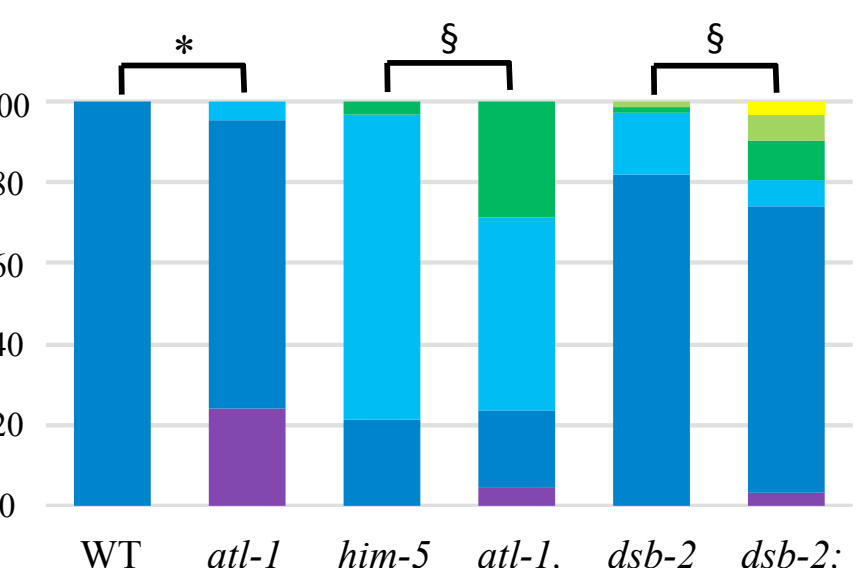

him-5

(28)

(21)

(66)

atl-1

(32)
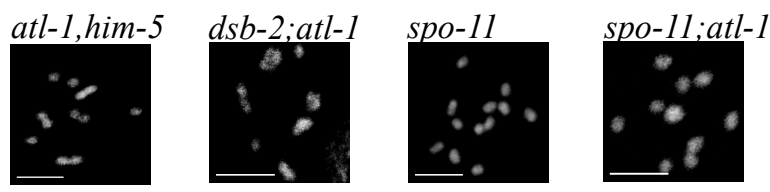

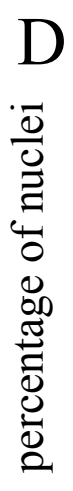
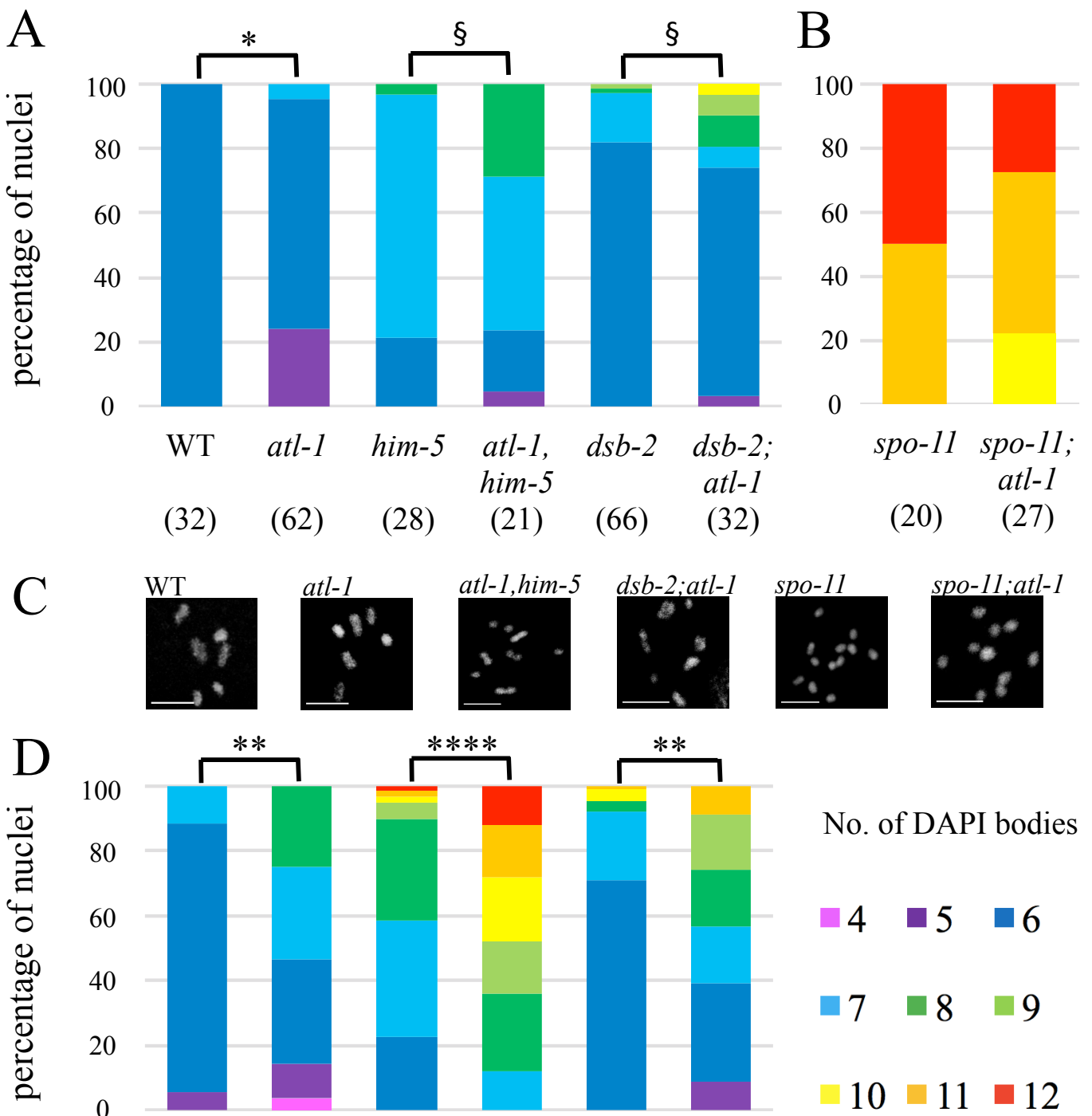

No. of DAPI bodies

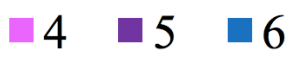

$\square 7 \quad \square \quad \square 9$

$10 \square 11 \square 12$
100

80

60

40

20

0

atm-1 atm-1; atm-1; atm-1; atm-1; atm-1; atl-1 him-5 atl-1, dsb-2 dsb-2; him-5

(86)

atl-1

(23)

Figure 6. atl-1 mutant animals exhibit defects in $\mathrm{CO}$ formation in wild-type and DSB-limiting situations. (A, B, D). Quantification of DAPI bodies in -1 nuclei containing the indicated number of DAPI bodies. $* \mathrm{P}<0.05, * * \mathrm{P}<0.01, * * * * \mathrm{P}<0.0001$, two-tailed Mann-Whitney test. $\S \mathrm{P}<0.05$, Fisher's exact test. (A) atl-1 has an increase in univalents alone or in combination with him-5 and $d s b-2$. (B) atl-1 mutants increase bivalents in spo-11, indicating a substantial amount of carry-though DNA damage from the mitosis and meiotic S phase. (C) Representative diakinesis-stage nuclei of different genotypes showing normal karyotype (wt) and mutant background with different proportions of bivalents and univalents. (D) Quantification of DAPI bodies in atm-1;atl-1 double mutants alone or with DSB-defective mutations shows synergistic effects from the loss of both gene functions. 


\section{FIGURE 7}

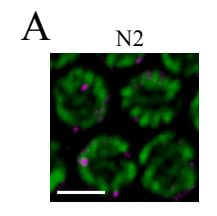

B
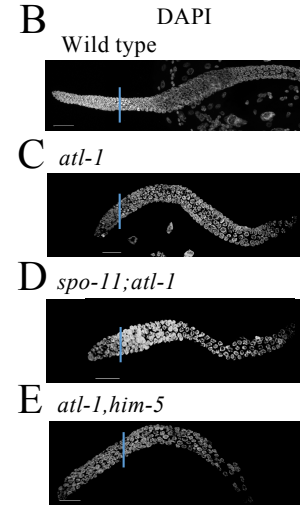

F $\operatorname{him}-5$

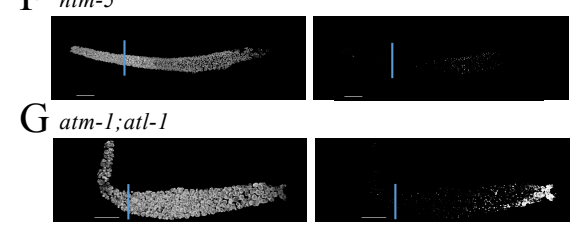

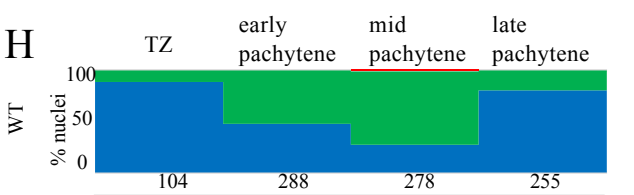
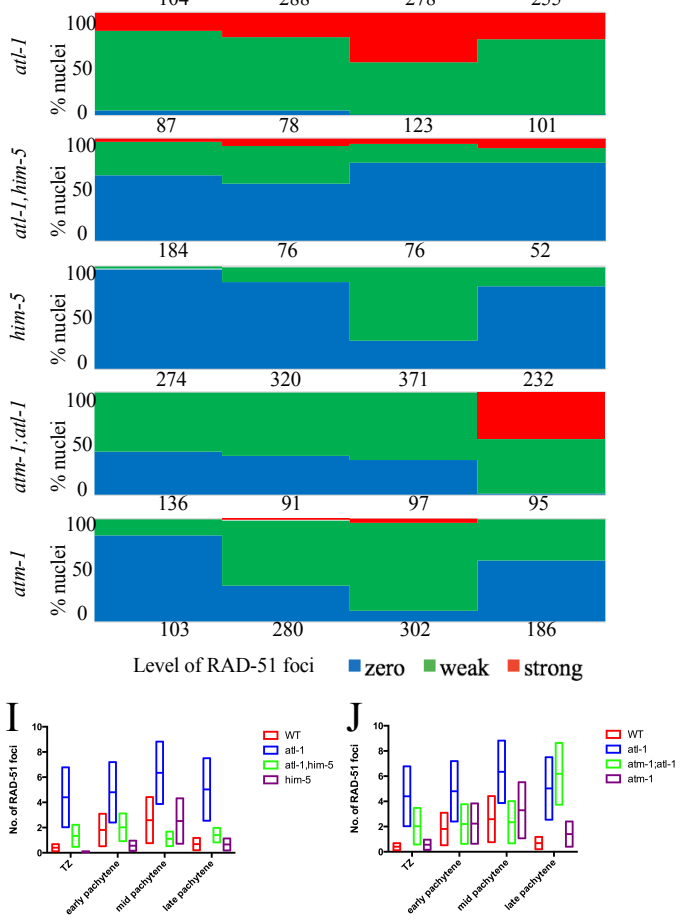

Figure 7. RAD-51 loading is altered by atl-1. (A) RAD-51 staining in pachytene nuclei of wild type $\mathrm{N} 2$, atl-1, and atl-1 him-5. RAD-51 (magenta); DAPI (green). Scale bar $=5 \mu \mathrm{m}$. The squares represent the three class of nuclei quantified in Figure 7C: Zero RAD-51 foci (blue); Weak staining (green); strong staining (red). (B-G) Representative images of DAPI and RAD-51 stained gonads from one-day old adults of indicated genotype. (H) Proportions of nuclei containing zero (blue), 1-6 (green, weak), or $>6$ foci (red, strong) RAD-51 foci in the leptotene-pachytene regions of the germ line as described in Materials and Methods. Numbers indicate total number of nuclei counted for each region for at least 3 germ lines/ genotype. (I, J) Range and average of RAD-51 foci for germ lines quantified in (H) for shown genotypes. 


\section{FIGURE 8}

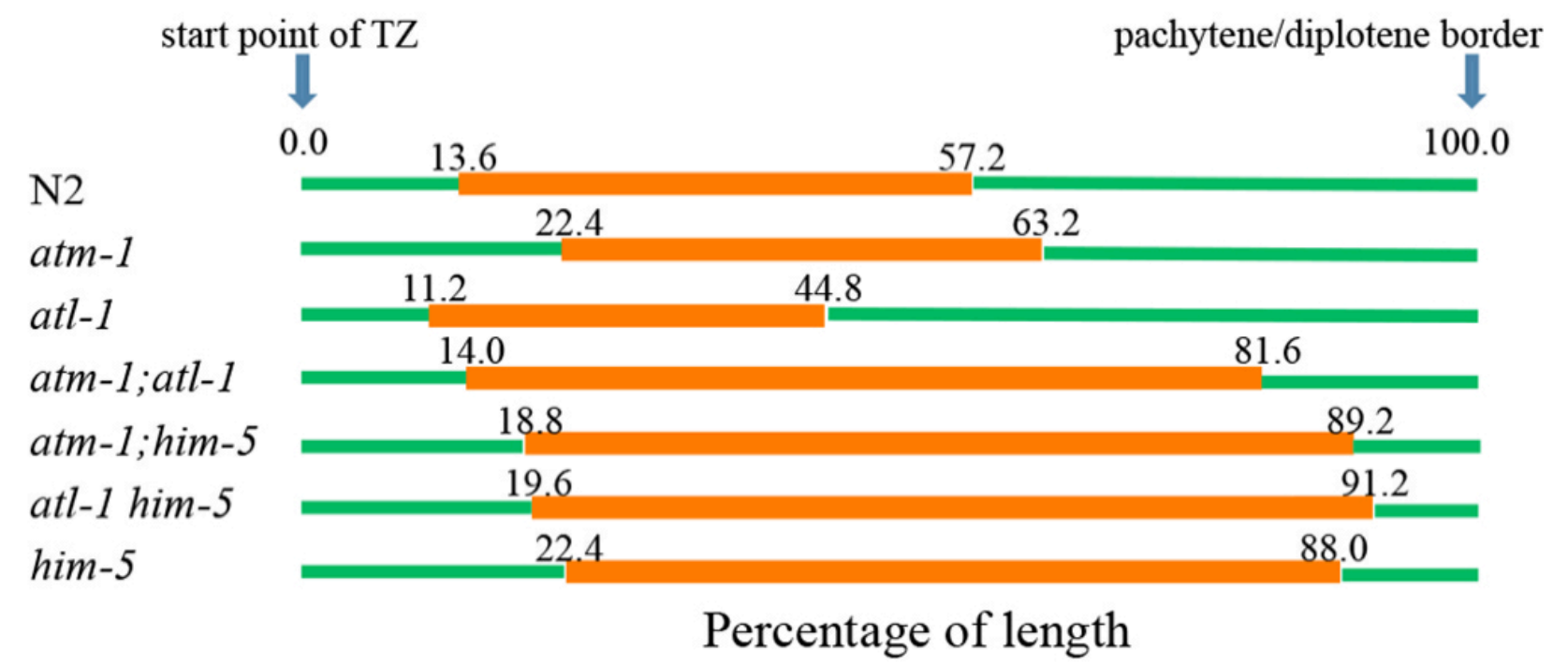

Figure 8. DSB-2 staining manifests different kinetics in the absence of ATM-1 or ATL-1 functions. Proportion of length of the germline region from meiotic onset to the pachytene/diplotene border that stain positive for nuclear localized DSB-2. Non-stained regions (green); stained (red). Genotypes are shown to the left with the average DSB-2 region depicted in orange $(\mathrm{n}=3,6,7,4,4,5,6$ for $\mathrm{N} 2$, atm-1, atl-1, atm-1; atl-1, atm-1; him-5, atl-1 him-5, and him-5, respectively). 


\section{FIGURE 9}

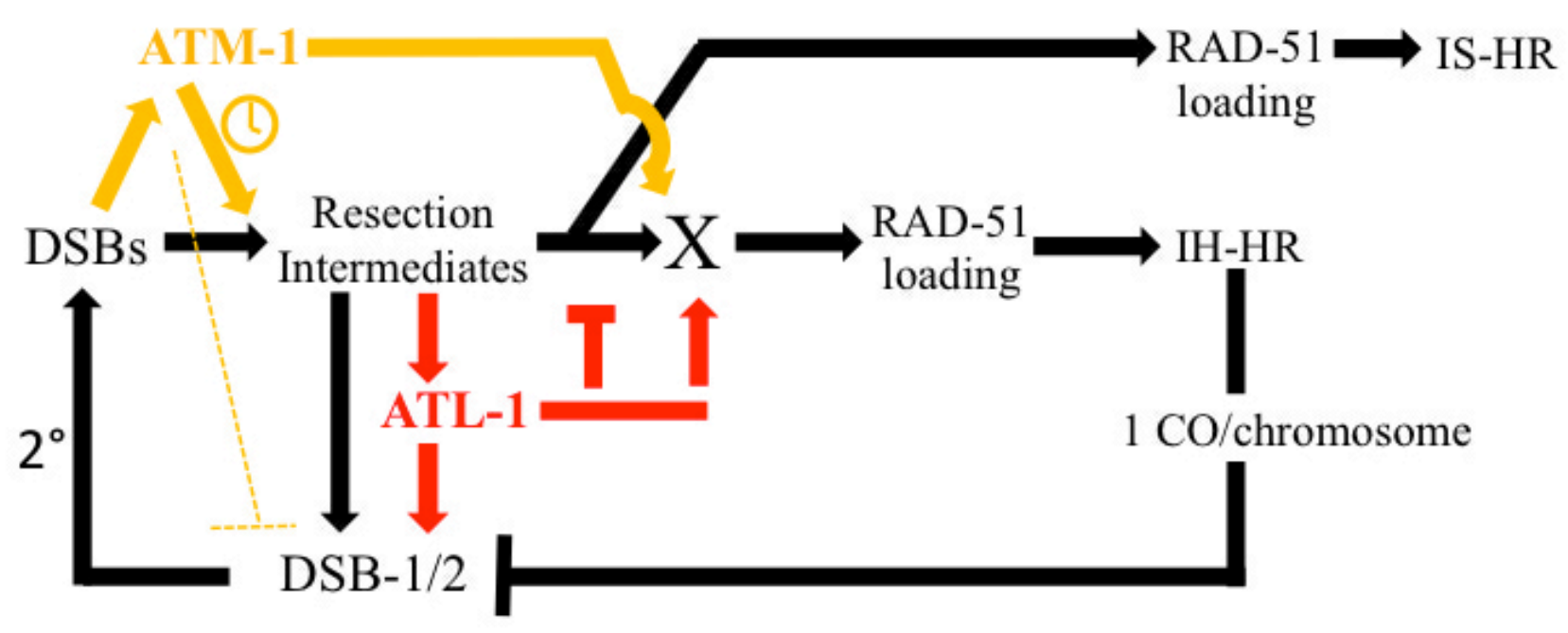

Figure 9. Model for ATM-1 and ATL-1 functions during meiosis. We propose that ATM-1 is activated by DSBs and then promotes the timely resection of ends and repair using the homolog as a repair template. The excess RAD-51 foci in atm-1 mutants could be explained if ATM-1 inhibits DSBs (dotted line), perhaps through DSB-1, but possibly SPO-11 or other accessory factors. Alternatively, delayed resection and IH-CO formation could keep the feed-forward loop active longer to allow additional DSBs to be made. We further posit that ATL-1 is activated by resection intermediates and both ATL-1 and a subset of pre-RAD-51 repair intermediates feed forward to promote additional DSB formation. ATL-1 then inhibits extensive resection and redundantly tp ATM-1 helps to promote RAD51 loading. These two activities can explain the delayed, excessive RAD-51 loading in atm-1; atl-1 double mutants. 